\title{
The impact of satellite constellations on space as an ancestral global commons
}

\author{
Aparna Venkatesan $\oplus^{1 凶}$, James Lowenthal ${ }^{2}$, Parvathy Prem $\oplus^{3}$ and Monica Vidaurri ${ }^{4,5}$
}

\begin{abstract}
Near-Earth space is becoming increasingly privatized, with the number of satellites in low-Earth orbits predicted to grow dramatically from about 2,000 at present to over 100,000 in the next decade due to the launch of planned satellite constellations. In addition to their direct impact on astronomy, the manner and pace of 'occupying' near-Earth space raises the risk of repeating the mistakes of colonization on a cosmic scale. We must consider the impact of satellite constellations, and related future initiatives, on the essential human right to dark skies and on cultural sky traditions across all peoples. We must also include all stakeholders for near-Earth space in the process of developing new policies for space treaties and planetary protection, the consequences of which will reach far beyond this century. We advocate for a radical shift in the policy framework of international regulatory bodies towards the view of space as an ancestral global commons that contains the heritage and future of humanity's scientific and cultural practices.
\end{abstract}

$\mathrm{N}$ ear-Earth space is becoming increasingly privatized and industrialized, with many consequences for science and humanity. In particular, the number of satellites in low-Earth orbit is predicted to grow dramatically from a couple of thousand at present to many tens of thousands in the near future due to the launch of satellite constellations planned by SpaceX, OneWeb, Amazon and other private companies. Each month of this year alone has brought headlines of another few hundred to tens of thousands satellites being approved by the Federal Communications Commission (FCC) for launch, confirming that an astounding 100,000 satellites in the sky by 2030 is not just feasible but quite likely.

These developments are not without benefits to a number of fields. The latest generation of low-cost small satellites, especially the picosatellites or CubeSats (mass $\sim 0.1-1 \mathrm{~kg}$ ), have already proven very useful for scientific research given how inexpensive and flexible they are (see ref. ${ }^{1}$ in this issue). CubeSats have the added advantage of rapid construction with off-the-shelf components. NASA's Heliophysics Division has made use of many CubeSats in the last six years, and the Planetary Science Division at NASA plans to utilize CubeSats in upcoming missions that are being developed. The Committee on Space Research has a task group on establishing a constellation of small satellites with the goal of being as internationally inclusive as possible, making inexpensive CubeSats an important tool for broadening participation and accessibility.

Nevertheless, the rapid increase in satellite constellations is a simmering crisis that is silently approaching the point of no return, and one that is easily missed in a year with so many crises. 2020 has revealed the escalating impact of pandemics like COVID-19 and climate change-from wildfires to floods to hurricanes-as well as the more long-standing pandemics impacting health such as systemic racism and economic inequality. The fallout from each of these crises has been borne disproportionately by the same communities. Collectively, these have revealed our vulnerabilities as a species and that we are at a crossroads as a planet. The paradigms and structural inequalities that led to this mega-crisis year are also behind how space is literally being occupied, with little coordinated international regulation, discussion of ethical considerations, or consensus-building from a shared vision for the future of humanity in space. The rush to claim near-Earth space is also leaving out the world's most minoritized communities, including Indigenous peoples, who need to be involved as stakeholders in decision-making.

The concerns around this escalating situation are broad and deep, and strike at the heart of our scientific and cultural relation to the night sky. Beyond our individual expertise and professional involvement with space, our identities as human beings and our relational view of space are also at stake. We discuss the growing scientific and cultural impact of satellite constellations, and related future initiatives for near-Earth space. We advocate for the regulation and protection of space as a shared community resource held in trust for future generations, much like air, water and land resources on Earth. This approach requires a radical shift in the policies of international regulatory bodies towards the view of space as an ancestral global commons that contains the heritage and future of humanity's scientific and cultural practices.

\section{Impact on astronomy}

The proliferation of low-Earth orbit satellites (LEOsats) at altitudes less than 2,000 km threatens our millennia-old ability to observe, discover and analyse the cosmos from the surface of the Earth. It is a special irony that a technology indebted to centuries of study of orbits and electromagnetic radiation from space now holds the power to prevent us permanently from further exploration of the Universe. It is an additional irony that astronomy-whose many institutions and facilities were built on the traditional lands of Indigenous peoplesis itself now threatened by the colonization of space by the satellite industry, and is seeking a seat at decision-making tables to mitigate the impact of LEOsats on astronomical research.

Satellite constellations could greatly improve communications and ongoing monitoring of Earth phenomena ranging from weather and climate to disaster management. Such large constellations also have the potential to offer global connectivity through

'Department of Physics and Astronomy, University of San Francisco, San Francisco, CA, USA. '2Department of Astronomy, Smith College, Northampton, MA, USA. ${ }^{3}$ Johns Hopkins University Applied Physics Laboratory, Laurel, MD, USA. ${ }^{4}$ Department of Physics and Astronomy, Howard University, Washington, DC, USA. ${ }^{5}$ NASA Goddard Space Flight Center, Greenbelt, MD, USA. $\varpi_{e}$-mail: avenkatesan@usfca.edu 
low-cost high-speed broadband internet. In principle, this could be the critical leap needed to bridge the very real digital divide ${ }^{2}$, especially for the world's most minoritized populations, including Indigenous communities. This divide has been exposed as a chasm during this pandemic year, affecting many millions of students and low-income workers. Broadband internet has become essential for daily life, especially during a pandemic year when remote forms of learning, teaching, work and even health (for example, telemedicine) have become the norm. In 2019, the FCC offered US\$20 billion in subsidies over ten years to address the digital divide in rural communities in the United States, which was quickly followed by a number of filings for LEOsats. LEOsat broadband may benefit rural communities more than urban areas-these 'last mile' connections are still challenging to complete relative to concentrated (urban) populations where ground-based cable/fibre internet infrastructure is cheaper. Large satellite constellations thus have the potential to bridge the digital chasm, but time will tell whether the promise of low-cost high-speed internet worldwide is achieved, and what the financial costs to customers are. This potential democratization of space is worth noting, even if it may not lead to fair participation in space.

A primary issue with commercial satellite constellations is how visible they are from the ground, especially at dawn and dusk, or soon after launch. There are a few mitigating options available at present that industry and the scientific community can choose ${ }^{3}$. The leading contributing factor to light pollution from satellite constellations is the satellites' size, which is on the order of a metre. Besides making satellites as small as possible, other proposed solutions include steps to darken the satellites and to calculate satellite positions to understand observational 'avoidance zones' by time or location for astronomy ${ }^{4,5}$. Of course, the only way to completely avoid the negative impacts from LEOsat constellations is to stop launching them entirely, but this is an extreme step that is unlikely to occur.

The SATCON1 workshop, the first of several ongoing investigations by the international astronomical research community into the impacts posed by upcoming constellations of up to 100,000 LEOsats by the end of the decade, concluded that negative impacts to ground-based optical and infrared astronomy are unavoidable, affecting practically every facet of astronomy. This workshop's detailed report is now available online ${ }^{3}$. Images and videos (see Supplementary Video) of the sky marred by bright streaks from SpaceX Starlink satellites, including images from professional observatories, circulated widely during the first year of launches, when only a few hundred (of more than 4,000 planned) satellites were in orbit. More recently, a few dozen stacked images of Comet Neowise this summer revealed numerous satellite constellation trails. We show a similar image of the southern sky at dusk in Fig. 1, which has streaks from Starlink satellites. Early simulations of, for example, 12,000 satellites by Michael Vlasov-far fewer than planned in the next few years-indicate a disastrously altered night sky. Large LEOsat constellations swarming the sky will be on a collision course with the capability of new legacy surveys to image deep and wide, whether at dark sites, twilight, the horizon or high Galactic latitudes ${ }^{3,6,7}$.

Some of the mitigating options presented earlier-such as darkening satellites and pinpointing their moving positions in the sky so telescopes can dodge them-require close cooperation between commercial satellite companies and the scientific community. SpaceX, the parent company of the Starlink LEOsat constellation, has been very cooperative in working with researchers, devoting significant resources towards finding solutions to this crisis for astronomy. We hope all satellite operators follow their example, for the benefit of everyone involved in research, amateur astronomy and other practices requiring dark skies. There is however no guarantee of such cooperation, and no regulation to hold bad actors accountable. Radio astronomy has relied for nearly a century on international agreements that control the radio spectrum and protect some especially valuable radio bands for astronomy. This will be needed more than ever as increasing requirements for radio bandwidth for up- and downlinks to all the LEOsats generate interference at radio bandpasses ${ }^{8}$. In contrast, the optical and near-infrared sky is an open frontier, with no controls in place to guard against light pollution from satellites or other sources by private or government bodies.

A follow-up workshop, SATCON2, planned for early 2021, will aim to study and develop the international regulatory framework needed to control large LEOsat constellations and their impact on astronomy and the night sky. In the meantime, we await the publication of a report from the National Science Foundation (NSF) sponsored group JASON on satellite constellations, which has so far been conducted entirely behind closed doors. In addition, the organizers of the Dark and Quiet Skies for Science and Society conference, to be hosted online in October 2020 by the United Nations Office for Outer Space Affairs, the International Astronomical Union, and the Instituto de Astrofísica de Canarias, hope that this conference's recommendations for the protection of the night sky for astronomy, human heritage and the natural world will be adopted by the United Nations General Assembly. These efforts are complemented by recent initiatives by over 40 scientific professional societies, including the American Astronomical Society, to develop 'civic science'. Civic science combines citizen science with civic engagement, and critical examination of our research to ask if it is ethical, mutually beneficial to and inclusive of the community in which it is being conducted.

Lastly, with the new generation of LEOsats, we are moving quickly towards a reality described by the 'Kessler syndrome', in which a cascading series of high-speed collisions could reduce thousands of satellites to an orbiting rubble pile in an impenetrable shell around the Earth, making LEOsat mega-constellations potentially their own worst enemy. The NASA Orbital Debris Program Office, in partnership with the US Department of Defense, tracks more than 500,000 objects in orbit with size larger than a marble, and all US satellite operators are required to file detailed collision avoidance plans before launching. With the dramatic increase in LEOsats, it is already becoming more difficult to safely launch future satellites through the increasingly congested space of LEOs. In contrast to the management of optical-infrared spectrum interference, space traffic management has many stakeholders, and there are many parties working on its regulation. Until a solution is reached, we are in danger of losing access to the sky.

\section{Treaties, space law and the uses of space}

The creation of the Outer Space Treaty (OST) in 1967 was a key milestone in the (at the time) new and growing human presence in space. The OST has many strengths, one of which was uniting humanity in shared knowledge as we began exploration of space as a new frontier. The use of celestial bodies and outer space for peaceful purposes, avoiding colonial competition and environmental disruption, and discouraging militarization and conflict in space are a few of the main premises of the OST, as well as the (far less widely ratified) Moon treaty. However, due to the non-binding nature of international law and lack of oversight or enforcement of the treaty, as well as the broad definitions of key components (such as 'colonial competition', 'militarization' and 'peaceful purposes'), it is easy to work around such premises through strategic loopholes.

As an example, military applications of space are still actively maintained and developed alongside satellite GPS and internet technologies, as well as suborbital military spaceflight. Additionally, although states are responsible for their own actions and liabilities in space, there is little by way of norm and policy with regard to private industry, including holding the private sector to similar 


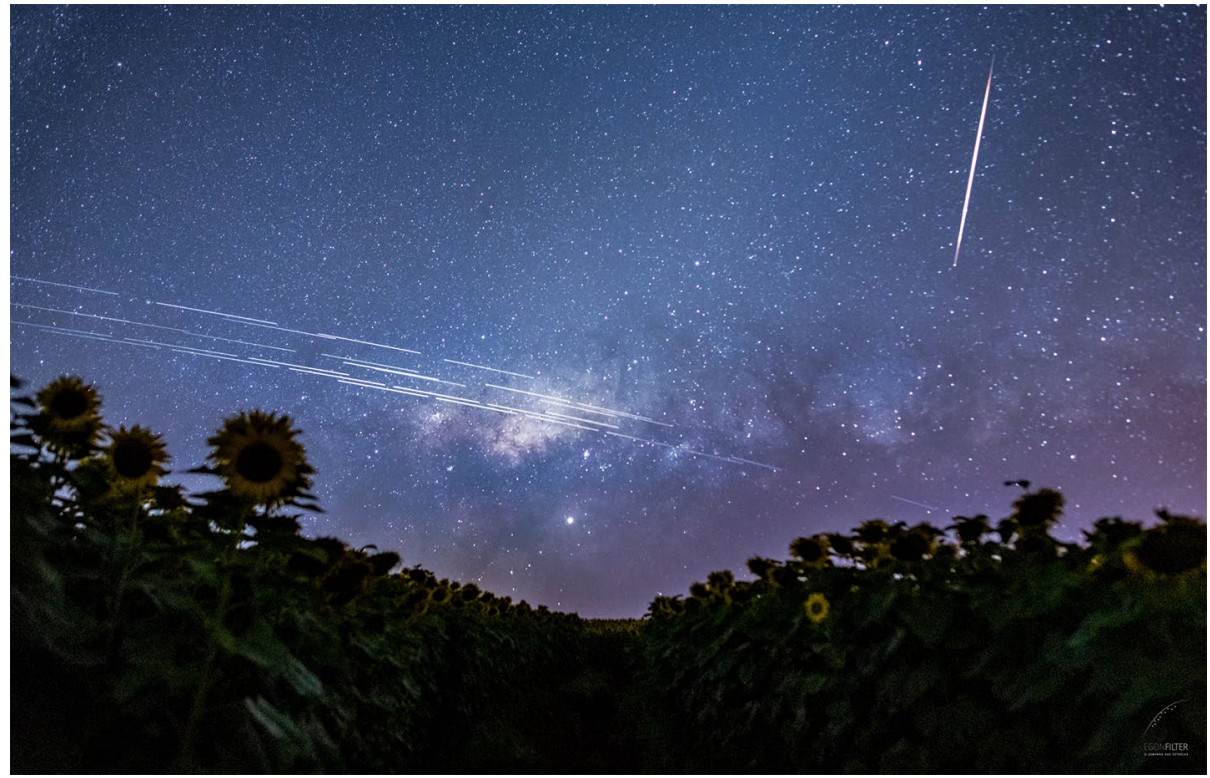

Fig. 1 | A view of the dusk sky over a sunflower farm in southern Brazil captures a number of passing Starlink satellites. This image is a composite of 33 stacked images with 13 seconds of exposure. Many astronomical studies can expect similar satellite trails in exposures of star fields, galaxies, and so on. The satellite trails here rival in brightness the meteor streak accidentally captured in the upper-right of the image. Credit: Egon Filter.

standards of operation, accountability and planetary protection as their respective states. When a payload of human DNA and tardigrades was spilled on the lunar surface without prior notification of the payload (see also ref. ${ }^{9}$ ), it highlighted two separate political questions. First, how does each space-faring entity view the importance of preserving non-Earth environments to the best of our ability, and so as not to offend any communities that view these celestial bodies as sacred, so that every nation or company may have equal access to space? And to what extent should domestic space policy be altered to regulate the activity of non-governmental actors in space to ensure that billionaires alone, for example, cannot alter the landscape of international relations in space according to their own activities? Although governments may not outwardly support goals or missions that would drastically alter current peaceful relations in space or any non-Earth environment, we are beginning to see (and will probably see far more) examples of the main components of the OST being ignored or actively worked around.

Given the disastrous history of Western colonization over the past few centuries on Earth and the widespread failure to honour land treaties with Indigenous and minoritized populations, perhaps the lack of compliance with, even the active working around of, the long-term thinking and humanistic goals of the OST and other space treaties are not a surprise. Like colonization, the rush to space is rooted in a survivalist scarcity mindset, leading to a first-come first-claim strategy to obtain and maintain an arbitrary economic and militaristic advantage that is 'on the clock'a rushed timeline unwarranted in its speed to stake, settle and mine, whether it's minerals and resources on Earth, asteroids and planetary environments ${ }^{10}$, or knowledge itself ${ }^{11}$. Although Earth and space resources are finite, and indeed the orbital space to even launch new satellites to low-Earth orbits is radically shrinking, revisiting the language and scope of space treaties from an abundance mindset is much needed, in the context of the sky we have all shared for millennia and from the perspective of space as a scientific and cultural resource for all of humanity. As Vidaurri et al. note, this requires factoring in ecological, environmental, Indigenous and ethical concerns for future space exploration and scientific missions in space ${ }^{12}$. These authors also call for "anti-colonial standards and protocol to ensure equal and fair participation in space", and for the astronomical community to prevent further ground-based colonialism.

Space is becoming the playground of billionaires ${ }^{13}$, and privately funded initiatives can often be nimbler and more responsive to evolving conditions and accelerated timelines. However, private actors in space are not accountable to the public (releasing records, self-assessments of science or diversity and inclusion outcomes). Federal and state agencies, despite their ponderous bureaucracy, do have accountability to the taxpayers, and to federal laws concerning, for example, harassment, racism, a hostile workplace culture, and so on-the human part of human environments that we will surely transport to lunar bases and beyond. Federal agencies can also distil key scientific goals from peer-reviewed publications and proposals, or decadal surveys-despite their limitations, this is closer to consensus-built goals than the projects of interest to private sponsors.

As public and private endeavours in space advance rapidly, an assessment and modernization of international space law and domestic space policy is inevitable. We advocate for an equally thorough discussion on the consideration of space as a human, protected environment. Space, including near-Earth orbits, has been used by humanity over its history for navigation, religious and cultural purposes, scientific study (rovers and human explorers), defence, and modern internet and other satellite telecommunications services. Although militarization, talk of colonization, and overall tension persists in the international space law landscape, our presence in space is ultimately a collaborative and peaceful endeavour. Disrupting the cooperative nature of space exploration is ultimately detrimental to the goals of any space actor or nation, especially in a landscape where survivability is low, accessible non-Earth environments are largely unknown and unexplored, and technological advances require global knowledge and coordinated international effort. Furthermore, the OST was created as the golden era of the space age was just beginning, when competitive ambition was high, resources on Earth and in space seemed limitless, and climate change and environmental concerns were factors on a far horizon. Today-in the language of the current COVID-19 pandemic-we have very different pre-existing conditions, making us deeply vulnerable to the many existential threats facing humanity, all species, and the planet itself. 
Thus, acknowledging space as a global commons (the notion that space is for all), while recognizing that our involvement in space may very well alter other environments and our own environment, requires a set of laws and policies that offer concrete accountability metrics, created in a shared ethics-minded space with the goal of compliance, while maintaining cooperation at an international level. These policies need not be a new endeavour: environmental laws, both international and domestic, have long been accountability-oriented while maintaining scientific collaboration and commercial access, and have even helped foster international cooperation. We must ask to whom space belongs, who truly has access to space, and who are the stakeholders for dark skies, celestial bodies and near-Earth space as we develop new policies.

\section{Planetary protection}

Like the environmental impact of satellite constellations, we must consider the short- and long-term effects of our exploration, related ethical considerations and how we alter near-Earth space and Solar System environments that we visit ${ }^{14}$. The key actors in developing and launching satellite constellations-whether nations or private corporations-are likely to play outsize roles in the future in accelerating human activities on the Moon, Mars and beyond.

Most regulation of planetary exploration to date has fallen under the purview of planetary protection, currently defined as "the practice of protecting solar system bodies from contamination by Earth life and protecting Earth from possible life forms that may be returned from other solar system bodies", which considers missions to other Solar System worlds in light of their implications for "understanding the process of chemical evolution or the origin of life".

In July 2020, NASA issued an interim directive (NID) titled 'Planetary Protection Categorization for Robotic and Crewed Missions to the Earth's Moon', designating most of the lunar surface as "not of direct interest for understanding the process of chemical evolution" (warranting no protection), with the exceptions of permanently shadowed regions (PSRs) near the lunar poles, and the Apollo landing and other "lunar historic" sites. This directive illustrates both the promise and limitations of current planetary protection policy. On the one hand the NID recognizes the scientific value of lunar PSRs, which are among the coldest places in the Solar System, and may hold a unique record of the delivery of water and other volatiles to the inner Solar System over the past several billion years. However, the only precaution that the NID deems necessary (perhaps limited by current planetary protection procedure) to protect this unique microenvironment is that "biological materials" carried on spacecraft be inventoried. This does not, for instance, mitigate concerns that the polar environment may be irrevocably altered by intensive human activity; the need to examine the potential impact of exhaust gases is important for the Moon as well as for other worlds in the Solar System ${ }^{15,16}$.

Science and commerce are only some aspects of human culture. The second exception carved out in the July 2020 NID, for sites of 'historical interest', raises the question of what we view as 'heritage', and how those views are shaped by dominant cultural paradigms. The preservation of one dominant thought system or heritage may be at the expense, or following the erasure, of another people's heritage. Earlier this year, NASA released the Artemis Accords, which aim to establish a set of shared principles for lunar surface operations. The accords have just been signed by seven countries, allowing state and private actors to proceed with resource extraction on the Moon including water ice at the lunar South Pole, raising concern among other countries as well as scientists about the gatekeeping of near-Earth space. One principle titled 'Protecting Heritage' includes language narrowly intended to protect the Apollo landing sites, a very specific type of heritage. There is no recognition that the lunar environment itself is worth protecting, or any acknowledgement of the cultural importance of the Moon. One might make the case that the cultural significance of the Moon-the brightest object in our night sky, our neighbour, and a witness to 4.5 billion years of Solar System history-is far greater than the boot-prints and detritus left behind on its surface just decades ago. We can make similar arguments about the heritage of space debris and artefacts or the deep significance of the night sky to many communities around the world, especially with intertwined scientific and cultural practices such as, for example, celestial navigation or wayfinding. Alternative approaches that view space as a commons may show us a promising way forward, including a recent call for considering space-based governance based on a New Zealand law, rooted in the indigenous Māori worldview, of granting legal personhood to natural resources ${ }^{17}$.

Contamination concerns with human exploration in space are often assigned to the purview of NASA's Office of Planetary Protection (OPP). Although human culture is a major component of space exploration, the OPP itself does not address these issues. The OPP is an advisory body with no real jurisdiction, and it solely concerns itself with matters regarding potential biocontamination and not greater environmental risks. The only required advisees are NASA missions, and the OPP is unable to handle matters regarding cultural concerns of celestial bodies. Broadening the power of the OPP to consider the environmental impact of non-Earth environments and to amend federal standards required by the National Environmental Policy Act are first steps ${ }^{18}$. In addition, planetary protection is very narrowly defined and considered at present, addressing just biocontamination. Planetary protection could be expanded to consider the ethical, cultural and legal considerations we have discussed, developed in a consensus-building process among all the affected constituents.

\section{Cultural practices with dark skies and space}

As we shared earlier, the role of satellite constellations, and, more broadly, the lack of consistent international regulation and consensus-building, strike at the core of humanity's scientific and cultural relation to the night sky, affecting millennia-old sky traditions and cultural practices across all nations around celestial cycles and the night sky ${ }^{19}$. Treating space as the 'Wild West' frontier that requires conquering continues to incentivize claiming by those who are well-resourced. It will also bring all the wounding and long-term consequences that imperial colonizing policies brought on Earthnow poised to be magnified on a cosmic scale for the most vulnerable minoritized communities on Earth, including Indigenous peoples.

Numerous Indigenous scholars and knowledge-holders have drawn attention to the multigenerational experiential wealth contained holistically in Indigenous knowledge $\mathrm{e}^{11,20,21,22}$, and its potential for creative solutions rooted in long-term thinking. As Leroy Littlebear notes in a series of talks hosted by the Indigenous Education Institute, Indigenous thought is relational, rooted in language, space and place, and based on renewal (sustainability). A key aspect of Indigenous identity and Indigenous knowledge is space referencing or 'Space and Place', learning in harmony with a specific time and place, which is part of a broader relational view of the Universe and our place in it $^{23,24}$. For example, in D/Lakota the relational view is epitomized by the often used phrase mitakuye oyasin, explained by Lakota elders as a philosophy that reminds everyone that we all come from one source and so need to respect each other to maintain wolakota or peace ${ }^{25}$. Numerous Indigenous and cultural practices around the world are based on this relational view of space, whether it is used for calendaring, sociocultural or religious events, agriculture and fishing cycles affecting food sovereignty, or celestial navigation or wayfinding. The Never Lost project and web resource created by Isabel Hawkins at the San Francisco Exploratorium in collaboration with the 'Imiloa Astronomy Center of Hawai'i showcases the breadth and depth of Polynesian navigation techniques; another excellent resource is the Polynesian Voyaging Society. 
These navigational practices specifically depend on the circumpolar skies or the relative rise and set locations of stars, constellations, the Moon or Sun, utilizing the horizon sky at dawn or dusk. The latter is already impacted by satellite constellations; more of the night sky will probably be affected in the near future as well. In addition, unlike some scientific projects that require long-term exposures and studies, cultural practices with the night sky are in real time and cannot utilize filters or software to remove low-Earth orbit satellite constellation trails and interference with observations.

A relational mindset is in contrast to our current 'rush to space, where a manufactured mandate of urgency from colonial policies ${ }^{26}$ is allowed to justify lack of consensus-building, relationships, long-term thinking, and access to decision-making power. Relationships, and relational views, are needed more than ever during crises. As Indigenous ecologist and knowledge-holder Robin Kimmerer notes in a recent talk, siloed practices and monoculture-based ways of thinking-as Western science has historically been-are not healthy or sustainable in the long run. Although many in the scientific community may not see the value of relational approaches to scientific projects, or may view science and culture as orthogonal concerns, we point out that sustainability and long-term thinking alone are pressing considerations for practically every scientific discipline including studies of climate change. In addition, physicists are used to seeming contradictions in the physical world-for example, we know and accept wave-particle duality at atomic scales, and also understand that one of these 'identities' is the forced outcome of a measurement or an incomplete non-integrative model. Such apparent paradoxes can coexist and are indeed at the heart of reality ${ }^{27}$. We advocate for a view of space that is similarly unified in science and culturewe know that science is inseparable from the cultural paradigms and values driving the science. The electron does not choose and neither should we.

Such a shift in how we view space requires consistent international regulatory policies and protocols that arise from a shared ethical space built in consensus, and from viewing dark skies as a human right from a commons that connects us all. This in turn requires beginning, not ending, with relationships and asking who the stakeholders in dark skies and space are, followed by soliciting their input in an equitable way so we can understand what fair participation in space might look like without appropriation or assimilation. This summer's racial justice protests have taught us that impersonal systems deliver the outcomes they were designed for, and can wound generations of individuals and communities. We cannot have a relationship with systems, only with the individuals in them. We need communities, not colonies, in our shared skies.

\section{Space as an ancestral global commons}

Most students of astrophysics learn early in their careers that we, and what we consume or use daily, have been in the cores of stars multiple times or created in the death throes of stars. When we analyse the data of galaxies from billions of light years away, we know we are looking at our cosmic past. This perspective-knowing that the Universe is within us and that we and the Sun will recycle back into future generations of stars and planets-is not as removed as some may believe from the relational view of many Indigenous cultures rooted in 'Space and Place', or cultural views of the night sky. Space is our past and our future; we are united in this ancestry and this ultimate fate.

We advocate for a radical shift in the policy framework of international regulatory bodies towards the view of space as an ancestral global commons that contains the heritage and future of humanity's scientific and cultural practices. We do not use the term radical lightly; this shift requires a profound change in attitude towards what space means to all of us and our inherent beliefs about human ownership of space. Such an attitude contradicts the policies of many nations and actors in space today; for example, as recently as April 2020, the White House issued an Executive Order asserting that "Outer space is a legally and physically unique domain of human activity, and the United States does not view it as a global commons".

We also urge federal and private space agencies and corporations to immediately establish a cultural ethics office that can offer an integrative approach for cultural intelligence, supporting scientific progress and cultural protocols from a shared ethical space rather than artificially siloed perspectives, and that the reports and findings of such offices be at the forefront of decision-making. This will begin the long overdue process of involving all the stakeholders for dark skies and near-Earth space, especially historically marginalized and Indigenous communities, as we develop new policies for space treaties and planetary protection that avoid replicating the costly mistakes of the past. The exhilaration of space exploration must be grounded in long-term thinking, centring of Indigenous voices, and sustainability.

As we grapple with an unprecedented scale and variety of crises in 2020, near-Earth space is being altered-quietly and permanently. What we do next with space, and for space, will reverberate for science and humanity for generations to come. We can choose to move away from a defensive transactional view of an inanimate space - that awaits ownership and extraction-to a more relational view of space as containing our scientific and cultural ancestry, a healthy ecosystem that holds scientific and cultural practices from all perspectives. Our understanding of our origins, as well as our collective future, in space depends on this.

Received: 7 September 2020; Accepted: 18 September 2020; Published online: 6 November 2020

\section{References}

1. Serjeant, S., Elvis, M. \& Tinetti, G. The future of astronomy with small satellites. Nat. Astron. https://doi.org/10.1038/s41550-020-1201-5 (2020).

2. Milazzo, M. P, Richey, C., Piatek, J., Vaughan, A. \& Venkatesan, A. The Growing Digital Divide and its Negative Impacts on NASA's Future Workforce (2020); https://go.nature.com/2S5zaIb

3. Walker, C. et al. Impact of Satellite Constellations on Optical Astronomy and Recommendations Toward Mitigations (NOIRLab, 2020); https://go.nature. com/30fAFbe

4. Hainaut, O. R. \& Williams, A. P. Impact of satellite constellations on astronomical observations with ESO telescopes in the visible and infrared domains. Astron. Astrophys. 636, A121 (2020).

5. McDowell, J. C. The low Earth orbit satellite population and impacts of the SpaceX Starlink constellation. Astrophys. J. Lett. 892, L36 (2020).

6. Massey, R., Lucatello, S. \& Benvenuti, P. The challenge of satellite megaconstellations. Nat. Astron. https://doi.org/10.1038/s41550-020-01224-9 (2020).

7. Levchenko, I., Xu, S., Wu, Y.-L. \& Bazaka, K. Hopes and concerns for astronomy of satellite constellations. Nat. Astron. https://doi.org/10.1038/ s41550-020-1141-0 (2020).

8. Hall, J. et al. Light pollution, radio interference, and space debris: threats and opportunities in the 2020s. Bull. Am. Astron. Soc. 51, 97 (2019).

9. Shahar, K. \& Greenbaum, D. Lessons in space regulations from the lunar tardigrades of the Beresheet hard landing. Nat. Astron. 4, 208-209 (2020).

10. Rivkin, A. S. et al. Asteroid Resource Utilization: Ethical Concerns and Progress (2020); https://go.nature.com/2SjvD9z

11. Kimmerer, R. W. Braiding Sweetgrass: Indigenous Wisdom, Scientific Knowledge and the Teachings of Plants (Milkweed Editions, 2015).

12. Vidaurri, M. et al. Absolute prioritization of planetary protection, safety, and avoiding imperialism in all future science missions: a policy perspective. Bull. Am. Astron. Soc. 51, https://baas.aas.org/pub/2020n7i276 (2019).

13. Venkatesan, A. et al. Towards inclusive practices with indigenous knowledge. Nat. Astron. 3, 1035-1037 (2019).

14. Tavares, F. et al. Ethical Exploration and the Role of Planetary Protection in Disrupting Colonial Practices (2020); https://go.nature.com/337LEFG

15. Prem, P., Hurley, D. M., Goldstein, D. B. \& Varghese, P. L. The evolution of a spacecraft-generated lunar exosphere. J. Geophys. Res. Planets 125, e06464 (2020).

16. Lorenz, R. D. Lander rocket exhaust effects on Europa regolith nitrogen assays. Planet. Space Sci. 127, 91-94 (2016). 
17. Tepper, E. \& Whitehead, C. Moon, Inc.: the New Zealand model of granting legal personality to natural resources applied to space. New Space 6, 288-298 (2018).

18. Vidaurri, M. \& Gilbert, A. Environmental Considerations in the age of Space Exploration: The Conservation and Protection of Non-Earth Environments (2020); https://go.nature.com/343ioPK

19. Penprase, B. E. The Power of Stars 2nd edn (Springer, 2017).

20. Maryboy, N. et al. The Cosmic Serpent: Collaboration with Integrity. Bridging Native Ways of Knowing and Western Science in Museums Settings (Indigenous Education Institute, 2012).

21. Cajete, G. Native Science: Natural Laws of Interdependence (Clear Light Publishers, 2000).

22. Lee, A. S., Rock, J., Wilson, W. \& Gawboy, C. The Red Day Star, the Women's Star and Venus: $\mathrm{D}(\mathrm{L} / \mathrm{N})$ akota, Ojibwe and other indigenous star knowledge. Preprint at https://arxiv.org/abs/2009.02127 (2020).

23. Venkatesan, A. \& Burgasser, A. Perspectives on the Indigenous worldviews in informal science education conference. Phys. Teach. 55, 456 (2017).

24. Venkatesan, A. et al. Collaboration with integrity: Indigenous knowledge in 21st century astronomy. Bull. Am. Astron. Soc. 51, https://baas.aas.org/ pub/2020n7i020/release/1 (2019).

25. Lee, A. S., Brummel, S., Ehret, K., Komperud, S. \& LaCoursiere, T. Building a framework for indigenous astronomy collaboration: native skywatchers, indigenous scientific knowledge systems, and the Bell Museum. Preprint at https://arxiv.org/abs/2008.07270 (2020).

26. Smith, L. T. Decolonizing Methodologies: Research and Indigenous Peoples (Zed, 2012).

27. Maryboy, N., Begay, D. H. \& Nichol, L. Paradox and transformation. Int. J. Appl. Sci. Sustain. Dev. 2, 15-24 (2020).

\section{Acknowledgements}

We dedicate this piece to the shared wealth of space as an ancestral global commons for humanity's scientific and cultural achievements and legacy. We express our profound gratitude to K. Krafton (AAS Bahcall Public Policy Fellow) whose invaluable expertise and around-the-clock availability for our questions were critical for this work. A.V. gratefully acknowledges her deeply sustaining work-life ecosystem, including her ancestors and her team of collaborators who model transformative partnerships between Indigenous and non-Indigenous knowledge systems: I. Hawkins, N. Maryboy, D. Begay, K. Kimura, L. Peticolas, A. Burgasser, D. Simons, A. Lee, L. Kimura, C. Baybayan, R. Wolfgramm, T. Wolfgramm, W. Kingi, C. Spiller, W. Barreno and J. Mucia. They have patiently taught and trusted her with their knowledge-keeping - their wisdom and stories are her medicine.

The views, thoughts, and opinions expressed in this article are those of the authors, and do not necessarily reflect the official policy or position of the authors' employers, or any other organization, agency, committee or other group or individual.

\section{Competing interests}

The authors declare no competing financial interests.

\section{Additional information}

Supplementary information is available for this paper at https://doi.org/10.1038/ s41550-020-01238-3.

Correspondence should be addressed to A.V.

Reprints and permissions information is available at www.nature.com/reprints.

Publisher's note Springer Nature remains neutral with regard to jurisdictional claims in published maps and institutional affiliations.

(c) Springer Nature Limited 2020 